\title{
Secondary Squamous Cell Carcinoma of the Oral Cavity after Nasopharyngeal Carcinoma
}

\section{Liyuan Dai, MD \\ Qigen Fang, MD \\ Peng Li, MD \\ Junfu Wu, MD \\ Xu Zhang, MS}

Department of Head Neck and Thyroid, Affiliated Cancer Hospital of Zhengzhou University, Henan Cancer Hospital, Zhengzhou, China
Correspondence: Qigen Fang, MD

Department of Head Neck and Thyroid, Affiliated Cancer Hospital of Zhengzhou

University, Henan Cancer Hospital, Zhengzhou,

Henan Province, PR China

Tel: 86-37165587239

Fax: 86-37165587998

E-mail: qigenfang@126.com

Received April 13, 2019

Accepted May 29, 2019

Published Online May 30, 2019

\section{Purpose}

The main goal of this study was to analyze the prognosis of secondary oral squamous cell carcinoma (SCC) with a comparison with sporadic oral SCC by a matched-pair design.

\section{Materials and Methods}

Records of patients with surgically treated primary oral SCC were reviewed, and a total of 83 patients with previous history of radiotherapy for nasopharyngeal carcinoma (NPC) were retrospectively enrolled. A matched-pair study was performed, each NPC survivor was matched with two sporadic oral SCC patients by age, sex, primary tumor site, adverse pathologic characteristics, disease stage, neck node status, and tumor stage. The overall survival (OS) and disease-specific survival (DSS) rates were calculated by the Kaplan-Meier method; independent prognostic factors were evaluated by the Cox proportional hazards method.

\section{Results}

Compared with sporadic oral SCC patients, NPC survivors were less likely to be smokers $(p=0.004)$, perineural invasion and lymphovascular invasion were more common in NPC survivors (both $p<0.001$ ). The 5-year OS and DSS rates in NPC survivors were $47 \%$ and $54 \%$, respectively; the 5-year OS and DSS rates in sporadic oral SCC patients were $62 \%$ and $67 \%$, respectively; the difference was significant (both $p<0.05$ ). In survival analysis, disease stage remained to be independent prognostic factor for both the OS and DSS.

\section{Conclusion}

NPC survivors had worse OS and DSS than sporadic oral SCC patients, NPC survivors were less likely to be smokers, but had higher opportunity of perineural invasion and lymphovascular invasion. Disease stage was the most important predictor for the survival in NPC survivors.

\section{Introduction}

Nasopharyngeal carcinoma (NPC) is a common malignant neoplasm in Asia, and the most effective treatment is radiotherapy, over $80 \%$ of NPC patients now have long term survival, and secondary malignancy is one of the major late complications [1,2]. Squamous cell carcinoma (SCC) is one of the most common secondary malignancies [3,4]. Only a few researchers have analyzed the oncologic outcome of second-

\section{Key words}

Nasopharyngeal carcinoma, Secondary squamous cell carcinoma, Radiotherapy, Oral squamous cell carcinoma, Head and neck squamous cell carcinoma 


\section{Materials and Methods}

\section{Patients and samples}

Medical records of consecutive patients with surgically treated primary oral SCC were reviewed between January 1985 to January 2019, enrolled patients must meet the following criteria: there was prior history of radiotherapy for NPC, and the latency between the diagnosis of oral SCC and the end of radiotherapy was not less than 3 years $[13,14]$. Information regarding age, sex, operation record, treatment, and follow-up was collected and analyzed. All the pathologic sections were re-evaluated and patients were re-staged by the 7th edition American Joint Committee on Cancer classification.

To compare the survival between NPC survivors and sporadic oral SCC patients, patients with sporadic oral SCC were also reviewed during the same study period, each NPC survivor was matched with two sporadic oral SCC patients, the matching process was performed by age ( \pm 5 years), sex, primary tumor site, perineural invasion, lymphovascular invasion, smoker, disease stage (stage I/II vs. stage III/IV), neck node status (positive vs. negative), and tumor stage (T1/T2 vs. T3/T4) [11]. Perineural invasion was considered to be present if tumor cells were identified within the perineural space and / or nerve bundle, lymphovascular infiltration was positive if tumor was noted within the lymphovascular channels [15]. Drinkers were defined as those who consumed at least one alcoholic drink per day for at least 1 year $[11,16]$, smokers were defined as those smoked on a daily basis or had quit smoking for less than 5 years [11].

\section{Statistical analysis}

The chi-square test was used to compare the demographic and clinical pathologic variables between the two groups. In survival analysis, variables of age, sex, perineural invasion, lymphovascular invasion, node stage, tumor stage, disease stage, and disease grade were included. The main oncologic outcome of interests were overall survival (OS) and diseasespecific survival (DSS). The OS was calculated from the time of the oral SCC diagnosis until death or the last follow-up. The DSS was defined as the time from the oral SCC diagnosis to cancer-caused death or the last follow-up [5,6]. The KaplanMeier method was used to evaluate the OS and DSS rates. Factors which were significant in univariate analysis (logrank method) were then analyzed by Cox model analysis to identify the independent risk factors for the OS and DSS rates. A p $<0.05$ was considered significant, and all statistical analyses were performed with SPSS ver. 20.0 (IBM Corp., Armonk, NY).

\section{Ethical statement}

The Zhengzhou University institutional research committee approved our study, and all participants provided written informed consent for medical research prior to initial treatment, and all experiments were performed in accordance with relevant guidelines and regulations.

\section{Results}

A total of 83 NPC survivors (67 male and 16 female) from 1430 patients with oral SCC were enrolled for analysis, the mean age was 63.4 years (range, 37 to 72 years). The mean latency from initial NPC to diagnosis of current oral SCC was 10.5 years (range, 4 to 32 years). Among the 83 patients, 72 cases had received conventional radiotherapy for NPC, and 11 cases had received 3D conformal radiation therapy or intensity-modulated radiation therapy for NPC. The mean radiation dose for NPC was 64.7 Gy (range, 51.4 to 79.2 Gy).

Negative margin was achieved in all patients. Neck dissection was performed in all the 83 cases, including five cases of radical neck dissection, seven cases of modified radical neck dissection, and 77 cases of selective dissection. Pathologic neck metastasis was noted in 28 patients.

In the 83 NPC survivors, 54 patients were diagnosed with tongue SCC, six cases with SCC of the mouth floor, 13 cases of buccal SCC, and 10 cases of lower gingiva SCC. During the same study period, 1,158 sporadic SCC patients of the tongue, mouth floor, buccal, and lower gingiva were also included for comparing. Comparison of demographic and clinical pathologic variables between NPC survivors and sporadic oral SCC patients was presented in Table 1. NPC survivors were less likely to be smokers $(p=0.004)$, perineural invasion and lymphovascular invasion were more common in NPC survivors than sporadic oral SCC patients (both $\mathrm{p}<$ 0.001). No significant difference regarding age, sex, and other adverse pathologic characteristics as well as adjuvant treatment was noted between the two groups (all $p>0.05$ ).

In NPC survivors, the mean follow-up time was 60.7 months (range, 9 to 221 months), 50 patients died, and in these 50 patients, 36 cases died of the disease, the 5-year OS and DSS rates were $47 \%$ and $54 \%$, respectively. After being matched (Table 2), in sporadic oral SCC group, the mean follow-up time was 70.1 months (range, 8 to 264 months), 66 patients died, and 50 cases died of the disease, the 5-year OS and DSS rates were $62 \%$ and $67 \%$, respectively. The two groups had significantly different 5-year OS and DSS rates (both $\mathrm{p}<0.05)$ (Figs. 1 and 2).

In further analysis of predictors for the OS in NPC sur- 
Table 1. Demographic and clinical pathologic information between NPC survivors and sporadic oral SCC patients

\begin{tabular}{|c|c|c|c|}
\hline & NPC survivor $(n=83)$ & Sporadic oral SCC $(n=1,158)$ & p-value \\
\hline \multicolumn{4}{|l|}{ Age (yr) } \\
\hline$\geq 60$ & 46 & 632 & 0.881 \\
\hline$<60$ & 37 & 526 & \\
\hline \multicolumn{4}{|l|}{ Sex } \\
\hline Male & 67 & 891 & 0.428 \\
\hline Female & 16 & 267 & \\
\hline \multicolumn{4}{|l|}{ Drinker } \\
\hline Yes & 8 & 200 & 0.072 \\
\hline No & 75 & 958 & \\
\hline \multicolumn{4}{|l|}{ Smoker } \\
\hline Yes & 11 & 323 & 0.004 \\
\hline No & 72 & 835 & \\
\hline \multicolumn{4}{|l|}{ Tumor site } \\
\hline Tongue & 54 & 486 & 0.004 \\
\hline Mouth floor & 6 & 312 & \\
\hline Buccal & 13 & 203 & \\
\hline Lower gingiva & 10 & 157 & \\
\hline \multicolumn{4}{|l|}{ Tumor stage } \\
\hline $\mathrm{T} 1+\mathrm{T} 2$ & 59 & 736 & 0.167 \\
\hline $\mathrm{T} 3+\mathrm{T} 4$ & 24 & 422 & \\
\hline \multicolumn{4}{|l|}{ Disease stage } \\
\hline $\mathrm{I}+\mathrm{II}$ & 51 & 633 & 0.230 \\
\hline III+IV & 32 & 525 & \\
\hline \multicolumn{4}{|l|}{ Node stage } \\
\hline N0 & 55 & 789 & 0.724 \\
\hline $\mathrm{N}+$ & 28 & 369 & \\
\hline \multicolumn{4}{|l|}{ Differentiation } \\
\hline Well & 47 & 554 & 0.133 \\
\hline Moderate & 23 & 377 & \\
\hline Poor & 13 & 167 & \\
\hline Unknown & 0 & 60 & \\
\hline \multicolumn{4}{|c|}{ Perineural invasion } \\
\hline Positive & 23 & 130 & $<0.001$ \\
\hline Negative & 60 & 949 & \\
\hline Unknown & 0 & 79 & \\
\hline \multicolumn{4}{|c|}{ Lymphovascular invasion } \\
\hline Positive & 16 & 153 & $<0.001$ \\
\hline Negative & 67 & 955 & \\
\hline Unknown & 0 & 50 & \\
\hline \multicolumn{4}{|l|}{ Treatment } \\
\hline S & 15 & 145 & 0.235 \\
\hline $\mathrm{S}+\mathrm{R}$ & 30 & 369 & \\
\hline $\mathrm{S}+\mathrm{R}+\mathrm{C}$ & 38 & 612 & \\
\hline
\end{tabular}

NPC, nasopharyngeal carcinoma; SCC, squamous cell carcinoma; S, surgery; R, radiotherapy; C, chemotherapy.

vivors, the factors of smoker, tumor site, disease stage, node stage, and differentiation were significantly associated with the OS in univariate analysis, further Cox model confirmed the independence of smoker, disease stage, and differentia- tion in predicting the OS (Table 3).

In further analysis of predictors for the DSS in NPC survivors, the factors of smoker, tumor stage, disease stage, perineural invasion, and treatment were significantly associated 
Table 2. Information comparison between NPC survivors and matched group

\begin{tabular}{|c|c|c|c|}
\hline & NPC survivor $(n=83)$ & Matched group ( $n=166)$ & p-value \\
\hline \multicolumn{4}{|l|}{ Age (yr) } \\
\hline$\geq 60$ & 46 & 88 & 0.719 \\
\hline$<60$ & 37 & 78 & \\
\hline \multicolumn{4}{|l|}{ Sex } \\
\hline Male & 67 & 134 & $>0.99$ \\
\hline Female & 16 & 32 & \\
\hline \multicolumn{4}{|l|}{ Drinker } \\
\hline Yes & 8 & 25 & 0.234 \\
\hline No & 75 & 141 & \\
\hline \multicolumn{4}{|l|}{ Smoker } \\
\hline Yes & 11 & 22 & $>0.99$ \\
\hline No & 72 & 144 & \\
\hline \multicolumn{4}{|l|}{ Tumor site } \\
\hline Tongue & 54 & 108 & $>0.99$ \\
\hline Mouth floor & 6 & 12 & \\
\hline Buccal & 13 & 26 & \\
\hline Lower gingiva & 10 & 20 & \\
\hline \multicolumn{4}{|l|}{ Tumor stage } \\
\hline $\mathrm{T} 1+\mathrm{T} 2$ & 59 & 118 & $>0.99$ \\
\hline $\mathrm{T} 3+\mathrm{T} 4$ & 24 & 48 & \\
\hline \multicolumn{4}{|l|}{ Disease stage } \\
\hline $\mathrm{I}+\mathrm{II}$ & 51 & 102 & $>0.99$ \\
\hline III+IV & 32 & 64 & \\
\hline \multicolumn{4}{|l|}{ Node stage } \\
\hline N0 & 55 & 110 & $>0.99$ \\
\hline $\mathrm{N}+$ & 28 & 56 & \\
\hline \multicolumn{4}{|l|}{ Differentiation } \\
\hline Well & 47 & 92 & 0.891 \\
\hline Moderate & 23 & 44 & \\
\hline Poor & 13 & 30 & \\
\hline \multicolumn{4}{|c|}{ Perineural invasion } \\
\hline Positive & 23 & 46 & $>0.99$ \\
\hline Negative & 60 & 120 & \\
\hline \multicolumn{4}{|c|}{ Lymphovascular invasion } \\
\hline Positive & 16 & 32 & $>0.99$ \\
\hline Negative & 67 & 134 & \\
\hline \multicolumn{4}{|l|}{ Treatment } \\
\hline S & 15 & 22 & 0.601 \\
\hline $\mathrm{S}+\mathrm{R}$ & 30 & 64 & \\
\hline $\mathrm{S}+\mathrm{R}+\mathrm{C}$ & 38 & 80 & \\
\hline
\end{tabular}

NPC, nasopharyngeal carcinoma; S, surgery; R, radiotherapy; C, chemotherapy.

with the DSS in univariate analysis, further Cox model confirmed the independence of disease stage, perineural invasion, and treatment in predicting the DSS (Table 4).

\section{Discussion}

Secondary oral SCC was relatively uncommon after radiotherapy for NPC with an incidence of less than 5\% [5-8, 17-19]. Very few researchers had analyzed the prognosis of oral SCC in NPC survivors. Sun et al. [7] retrospectively ana- 


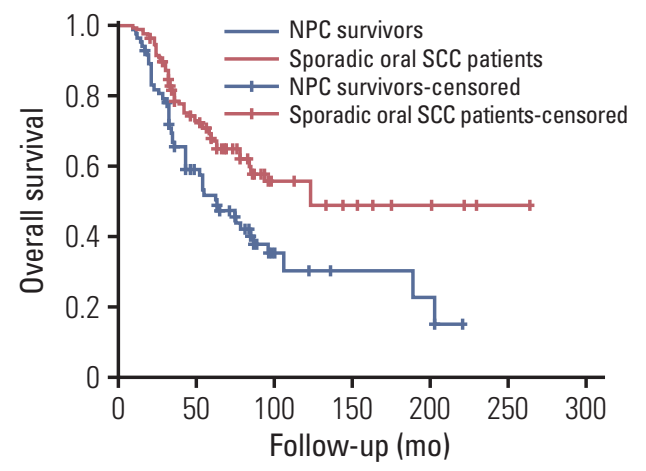

Fig. 1. Overall survival rates between nasopharyngeal carcinoma (NPC) survivors and matched sporadic oral squamous cell carcinoma (SCC) patients $(\mathrm{p}=0.001)$.

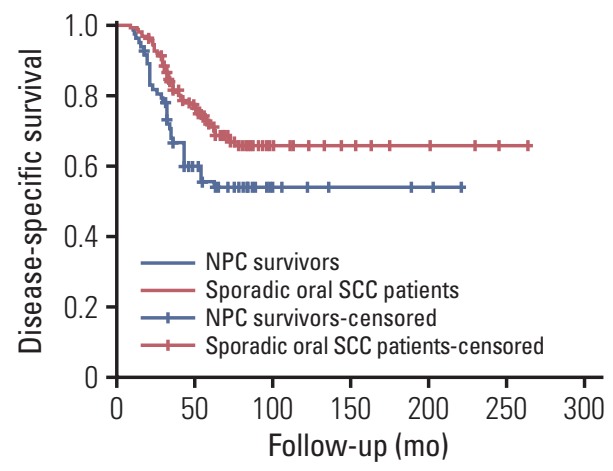

Fig. 2. Disease-specific survival rates between nasopharyngeal carcinoma (NPC) survivors and matched sporadic oral squamous cell carcinoma (SCC) patients $(\mathrm{p}=0.017$ ).

Table 3. Prognostic factors for the overall survival in NPC survivors

\begin{tabular}{|c|c|c|c|}
\hline \multirow{2}{*}{ Variable } & \multirow{2}{*}{$\begin{array}{l}\text { Univariate } \\
\text { Log-rank test }\end{array}$} & \multicolumn{2}{|c|}{ Cox model } \\
\hline & & HR $(95 \% \mathrm{CI})$ & p-value \\
\hline Age ( $\geq 60$ yr vs. $<60$ yr $)$ & 0.358 & - & - \\
\hline Sex (male vs. female) & 0.288 & - & - \\
\hline Drinker (yes vs. no) & 0.112 & - & - \\
\hline Smoker (yes vs. no) & 0.025 & $1.963(1.275-4.002)$ & 0.031 \\
\hline Tumor site (tongue vs. others) & 0.034 & $2.111(0.687-8.244)$ & 0.657 \\
\hline Tumor stage (T1+T2 vs. T3+T4) & 0.096 & - & - \\
\hline Node stage (N0 vs. N+) & 0.044 & $3.013(0.846-6.897)$ & 0.273 \\
\hline Disease stage (I+II vs. III+IV) & 0.008 & $3.482(1.852-10.336)$ & $<0.001$ \\
\hline Differentiation (well vs. moderate+poor) & 0.017 & $2.541(1.344-6.579)$ & 0.005 \\
\hline Perineural invasion (yes vs. no) & 0.324 & - & - \\
\hline Lymphovascular invasion (yes vs. no) & 0.119 & - & - \\
\hline Treatment (surgery alone vs. others) & 0.613 & - & - \\
\hline
\end{tabular}

NPC, nasopharyngeal carcinoma; HR, hazard ratio; CI, confidence interval.

lyzed 68 patients who had then developed tongue SCC after radiotherapy for NPC, the authors depicted eight of these patients had clinical lymph node metastasis, and 45 presented with stage I-II disease at the time of the diagnosis. Surgery or radiotherapy alone was an effective treatment for patients with stage I-II tongue SCC, but patients with stage III-IV disease had a poor prognosis. Song et al. [8] reported of the 62 included patients, 42 patients died, the 3- and 5-year OS rates were $41 \%$ and $30 \%$, respectively. Both two studies failed to compare the prognosis difference between NPC survivors and sporadic oral SCC patients. Zhang et al. [6] enrolled 73 patients with tongue SCC following radiotherapy, and the authors described that NPC survivors were less likely to have lymph node metastasis, but more likely to have a more advanced $\mathrm{T}$ classification, compared to sporadic tongue SCC patients, NPC survivors had worse OS but similar disease-free survival. Similar finding was reported regarding gingiva SCC by the same research team [5]. But in the above-mentioned two studies, the two groups had different tumor stage, node stage, and disease stage, all the variation could affect the comprehension of their outcome. The current study was firstly performed by a matched-pair design to shield the interfere of as many as confounding factors [20], it was noted NPC survivors had worse OS and DSS compared to sporadic oral SCC. At least the following three aspects called for consideration: in the one hand, there was negative prognostic effect of the previous radiotherapy on the survival of patients with oral SCC by prior researchers [21]. On the other hand, the difference in the immune status and the accumulation of genetic damage due to a history of 
Table 4. Prognostic factors for the disease-specific survival in nasopharyngeal carcinoma survivors

\begin{tabular}{|c|c|c|c|}
\hline \multirow{2}{*}{ Variable } & \multirow{2}{*}{$\begin{array}{c}\text { Univariate } \\
\text { Log-rank test }\end{array}$} & \multicolumn{2}{|c|}{ Cox model } \\
\hline & & HR $(95 \%$ CI) & $\overline{\text { p-value }}$ \\
\hline Age $(\geq 60$ yr vs. $<60$ yr $)$ & 0.147 & - & - \\
\hline Sex (male vs. female) & 0.638 & - & - \\
\hline Drinker (yes vs. no) & 0.241 & - & - \\
\hline Smoker (yes vs. no) & 0.013 & $1.951(0.847-3.003)$ & 0.287 \\
\hline Tumor site (tongue vs. others) & 0.378 & - & - \\
\hline Tumor stage (T1+T2 vs. T3+T4) & 0.016 & $2.631(0.411-6.387)$ & 0.612 \\
\hline Node stage (N0 vs. N+) & 0.188 & - & - \\
\hline Disease stage (I+II vs. III+IV) & 0.007 & $4.021(1.637-13.554)$ & $<0.001$ \\
\hline Differentiation (well vs. moderate+poor) & 0.087 & - & - \\
\hline Perineural invasion (yes vs. no) & 0.016 & $2.002(1.174-3.982)$ & 0.011 \\
\hline Lymphovascular invasion (yes vs. no) & 0.325 & - & - \\
\hline Treatment (surgery alone vs. others) & 0.023 & $1.988(1.164-3.863)$ & 0.013 \\
\hline
\end{tabular}

$\mathrm{HR}$, hazard ratio; $\mathrm{CI}$, confidence interval.

radiation could help explain this situation, in addition, the excess mortality due to NPC and severe late complications was also partially responsible [22,23].

Smoking was a common risk factor for the development of oral SCC, but less smokers were noted in NPC survivors in the current study. The finding might support the viewpoint that oral SCC in the NPC survivors and sporadic oral SCC might have different carcinogenesis [24], the sporadic oral SCC patients were more likely to be accompanied by mucosal field-cancerization, such as smoking. But in NPC survivors, the most possible explanation was the involvement of the bystander effect in cancer induction, it might induce the DNA impairment [25].

Perineural invasion and lymphovascular invasion were widely investigative predictors for poor prognosis in oral SCC patients, strongly correlating with tumor site, tumor stage, and disease stage [26,27]. It was noted perineural invasion and lymphovascular invasion were more frequent in NPC survivors, the finding was a little out of our expectation. In a previous paper published by Zhang et al. [6], compared to sporadic tongue SCC patients, NPC survivors tended to have a low risk of the worst pattern of invasion and low tumor budding, both the two markers were independent predictors for poor prognosis. No other similar literature was available for comparing, the current study was the first to find there is a significantly higher prevalence of perineural invasion and lymphovascular invasion in NPC survivors. Tissue damage and increased vascular osmotic fragility caused by the previous radiotherapy might be partially responsible for the finding. Another possible explanation was that there might be different gene mutation between the two groups, some authors had described a mutation in
BRCA1, BRCA2, and p53 was noted in patients' radiationassociated breast carcinoma [28], there might be a similar phenomenon in oral SCC, but it required more studies.

Prognostic factors for the survival in NPC survivors were rarely analyzed $[7,8]$. Song et al. [8] concluded the key prognostic factors influencing the survival were age when NPC was diagnosed, TNM stage, and surgery treatment modality. Multivariate analysis revealed that the clinical TNM stage was an independent risk factor for patient survival. Sun et al. [7] described the risk factors influencing the survival of these patients independently were recurrence of their NPC, use of alcohol, the clinical TNM stage, and the latency period in multivariate analysis. Similar finding was also noted in current research, moreover, other well known prognostic predictors in sporadic oral SCC were also confirmed in NPC survivors.

The main treatment for oral SCC was the combined therapy dominated by surgery. For NPC survivors, it was difficult to choose the right time and dose to conduct radiotherapy, as re-irradiation was tolerable and feasible in properly selected patients who have a radiation history, it might improve the survival [5]. Postoperative re-irradiation should be advised in the case that patients required more disease control at the expense of higher toxicity $[5,9]$.

All the literature regarding secondary head neck SCC including this study was retrospective or was described by demographic results; we hope the current research could provide assistance in learning this relatively uncommon cancer type and looking for better ways to analyze and control its progression.

Limitation of this study must be acknowledged: firstly, there was inherent bias in a retrospective study. Second, the 
sample size was relatively small, possibly reducing the statistical power, therefore, larger sample-size studies were needed to clarify the question.

In summary, NPC survivors had worse OS and DSS than sporadic oral SCC patients, NPC survivors were less likely to be smokers, but had a higher opportunity of perineural invasion and lymphovascular invasion. Disease stage was the most important predictor for the survival in NPC survivors.

\section{Conflicts of Interest}

Conflict of interest relevant to this article was not reported.

\section{References}

1. Lee HF, Lan JH, Chao PJ, Ting HM, Chen HC, Hsu HC, et al. Radiation-induced secondary malignancies for nasopharyngeal carcinoma: a pilot study of patients treated via IMRT or VMAT. Cancer Manag Res. 2018;10:131-41.

2. Oei RW, Ye L, Kong F, Du C, Zhai R, Xu T, et al. Prognostic value of inflammation-based prognostic index in patients with nasopharyngeal carcinoma: a propensity score matching study. Cancer Manag Res. 2018;10:2785-97.

3. Diallo I, Haddy N, Adjadj E, Samand A, Quiniou E, Chavaudra J, et al. Frequency distribution of second solid cancer locations in relation to the irradiated volume among 115 patients treated for childhood cancer. Int J Radiat Oncol Biol Phys. 2009;74:876-83.

4. Tubiana M. Can we reduce the incidence of second primary malignancies occurring after radiotherapy? A critical review. Radiother Oncol. 2009;91:4-15.

5. Fu X, Chen S, Chen W, Yang Z, Song M, Li H, et al. Clinical analysis of second primary gingival squamous cell carcinoma after radiotherapy. Oral Oncol. 2018;84:20-4.

6. Zhang P, Zhang L, Liu H, Zhao L, Li Y, Shen JX, et al. Clinicopathologic characteristics and prognosis of tongue squamous cell carcinoma in patients with and without a history of radiation for nasopharyngeal carcinoma: a matched case-control study. Cancer Res Treat. 2017;49:695-705.

7. Sun C, Hu Z, Zhong Z, Jiang Y, Sun R, Fei J, et al. Clinical and prognostic analysis of second primary squamous cell carcinoma of the tongue after radiotherapy for nasopharyngeal carcinoma. Br J Oral Maxillofac Surg. 2014;52:715-20.

8. Song M, Zhuang SM, Chen SW, Zhang Q, Yang AK, Wang LP, et al. Survival study and treatment strategy for second primary tumors in the oral cavity in patients with nasopharyngeal carcinoma after definitive radiation. Head Neck. 2012;34: 1551-5.

9. Strojan P, Corry J, Eisbruch A, Vermorken JB, Mendenhall WM, Lee AW, et al. Recurrent and second primary squamous cell carcinoma of the head and neck: when and how to reirradiate. Head Neck. 2015;37:134-50.

10. Fang Q, Li P, Qi J, Luo R, Chen D, Zhang X. Value of lingual lymph node metastasis in patients with squamous cell carcinoma of the tongue. Laryngoscope. 2019;129:2527-30.

11. Fang QG, Shi S, Liu FY, Sun CF. Squamous cell carcinoma of the oral cavity in ever smokers: a matched-pair analysis of sur- vival. J Craniofac Surg. 2014;25:934-7.

12. Fang QG, Shi S, Li M, Zhang X, Liu FY, Sun CF. Free flap reconstruction versus non-free flap reconstruction in treating elderly patients with advanced oral cancer. J Oral Maxillofac Surg. 2014;72:1420-4.

13. Cahan WG, Woodard HQ, Higinbotham NL, Stewart FW, Coley BL. Sarcoma arising in irradiated bone: report of eleven cases. 1948. Cancer. 1998;82:8-34.

14. Arlen M, Higinbotham NL, Huvos AG, Marcove RC, Miller T, Shah IC. Radiation-induced sarcoma of bone. Cancer. 1971;28: 1087-99.

15. Skulsky SL, O'Sullivan B, McArdle O, Leader M, Roche M, Conlon PJ, et al. Review of high-risk features of cutaneous squamous cell carcinoma and discrepancies between the American Joint Committee on Cancer and NCCN Clinical Practice Guidelines In Oncology. Head Neck. 2017;39:578-94.

16. Bachar G, Hod R, Goldstein DP, Irish JC, Gullane PJ, Brown $\mathrm{D}$, et al. Outcome of oral tongue squamous cell carcinoma in patients with and without known risk factors. Oral Oncol. 2011;47:45-50.

17. Kong L, Lu JJ, Hu C, Guo X, Wu Y, Zhang Y. The risk of second primary tumors in patients with nasopharyngeal carcinoma after definitive radiotherapy. Cancer. 2006;107:1287-93.

18. Chen MC, Feng IJ, Lu CH, Chen CC, Lin JT, Huang SH, et al. The incidence and risk of second primary cancers in patients with nasopharyngeal carcinoma: a population-based study in Taiwan over a 25-year period (1979-2003). Ann Oncol. 2008;19: 1180-6.

19. Zhao W, Lei H, Zhu X, Li L, Qu S, Liang X, et al. The clinical characteristics of secondary primary tumors in patients with nasopharyngeal carcinoma after intensity-modulated radiotherapy: A retrospective analysis. Medicine (Baltimore). 2016; 95:e5364.

20. Chen SW, Zhang Q, Guo ZM, Chen WK, Liu WW, Chen YF, et al. Trends in clinical features and survival of oral cavity cancer: fifty years of experience with 3,362 consecutive cases from a single institution. Cancer Manag Res. 2018;10:4523-35.

21. Paleri V, Wight RG, Silver CE, Haigentz M Jr, Takes RP, Bradley PJ, et al. Comorbidity in head and neck cancer: a critical appraisal and recommendations for practice. Oral Oncol. 2010;46:712-9.

22. Sanna G, Lorizzo K, Rotmensz N, Bagnardi V, Cinieri S, 
Colleoni M, et al. Breast cancer in Hodgkin's disease and nonHodgkin's lymphoma survivors. Ann Oncol. 2007;18:288-92.

23. Ng AK, Bernardo MP, Weller E, Backstrand KH, Silver B, Marcus $\mathrm{KC}$, et al. Long-term survival and competing causes of death in patients with early-stage Hodgkin's disease treated at age 50 or younger. J Clin Oncol. 2002;20:2101-8.

24. Teo PM, Chan AT, Leung SF, Chau RM, Yu PK, King WW, et al. Increased incidence of tongue cancer after primary radiotherapy for nasopharyngeal carcinoma: the possibility of radiation carcinogenesis. Eur J Cancer. 1999;35:219-25.

25. Bostrom PJ, Soloway MS. Secondary cancer after radiotherapy for prostate cancer: should we be more aware of the risk? Eur Urol. 2007;52:973-82.

26. Chatzistefanou I, Lubek J, Markou K, Ord RA. The role of per- ineural invasion in treatment decisions for oral cancer patients: a review of the literature. J Craniomaxillofac Surg. 2017;45: 821-5.

27. Sahu V, Nigam L, Agnihotri V, Gupta A, Shekhar S, Subbarao $\mathrm{N}$, et al. Diagnostic significance of p38 isoforms (p38alpha, p38beta, p38gamma, p38delta) in head and neck squamous cell carcinoma: comparative serum level evaluation and design of novel peptide inhibitor targeting the same. Cancer Res Treat. 2019;51:313-25.

28. Broeks A, Braaf LM, Wessels LF, van de Vijver M, De Bruin ML, Stovall M, et al. Radiation-associated breast tumors display a distinct gene expression profile. Int J Radiat Oncol Biol Phys. 2010;76:540-7. 